\title{
Comment on "Prevalence of arterial hypertension and associated factors: a population-based study"
}

\author{
Zi Wei Chen ${ }^{1} \oplus$, Chenchen Pan $^{1 *}$
}

Dear Editor,

We are more than glad to read the high-quality article entitled "Prevalence of arterial hypertension and associated factors: a population-based study," published by Layann ${ }^{1}$ and his research group ${ }^{1}$. They found that some factors, such as older, brown, sedentary, and overweight, are significantly associated with hypertension. However, as far as I am concerned, there are some questions that should be discussed further in the study.

First of all, the authors have not confirmed the discharge and inclusion criteria, and the source of the sample is still controversial. They should clarify the definition of overweight and obesity, as well as marital status. In addition, we do not know the start time and end time of the study.

As proposed in this study, the percentage of single subjects is lower than that of married or divorced subjects. The reason is failure to explain further in this study. The relationship between single subjects and divorced subjects, and whether hypertension is psychological or physiological need to be explained in detail. The authors also mentioned that hypertension has nothing to do with school. Two problems need to be explored in this case. First, the school will inherit the psychological pressure. Second, whether single or divorced, their psychological problems have the same effect. Therefore, the result is whether hypertension is related to single or divorced has nothing to do with school; this contradicts the result. Whether the study pressure degree of school is a comparative experiment is not described in detail.
The definition of overweight or obesity should be offered in this study. The authors found that hypertension is related to education level. There is no specific concept of education level, regarding comprehensive education level or education level of a certain kind of knowledge.

We are still in doubt whether the contradiction between skin color and hypertension-related complex conclusions mentioned in this article is due to the sample being too small or not representative, and therefore the authors should describe further in detail. In this study, the definition of sitting was not clear. In our views, strictly speaking, sitting was sedentary, not exercising, which is just long. Sedentary exercise is exercise after or before a long life.

Finally, the study discusses only adults, but not young children. The reason behind this should also be briefly explained. In addition to age, what makes children less prone to such diseases should be explained in order to guide the role of promoting adult prevention and reduction of such diseases.

\section{AUTHORS' CONTRIBUTIONS}

ZWC: Resources, Software, Supervision, Validation, Visualization, Writing - original draft, Writing - review \& editing. CP: Conceptualization, Data curation, Formal Analysis, Funding acquisition, Investigation, Methodology, Project administration.

\section{REFERENCE}

1. Lavôr LCC, de Sousa RR, Rodrigues LARL, Rodrigues Filho OS, Paiva AA, Frota KMG. Prevalence of arterial hypertension and associated factors: a population-based study. Rev Assoc Med Bras. (1992). 2020;66(5):630-6. https://doi.org/10.1590/1806-9282.66.5.630

${ }^{1}$ Taizhou University, School of Medicine - Taizhou, China.

*Corresponding author: chenchenpan1@126.com

Conflicts of interest: the authors declare there is no conflicts of interest. Funding: none.

Received on October 17, 2021. Accepted on October 30, 2021. 